\title{
Comparison of Isoflurane and Sevoflurane Anesthesia in Holstein Calves for Placement of Portal and Jugular Vein Cannulas
}

\author{
${ }^{1}$ Glen Sellers, ${ }^{1}$ Hui-Chu Lin, ${ }^{1}$ Manuel Felipe Chamorro and ${ }^{2}$ Paul Harold Walz \\ ${ }^{1}$ Department of Clinical Sciences, \\ ${ }^{2}$ Department of Pathobiology, \\ College of Veterinary Medicine, Auburn University, Auburn, Alabama, 36849, USA
}

Received 2013-02-28, Revised 2013-03-14; Accepted 2013-03-16

\begin{abstract}
Isoflurane and sevoflurane are the two most commonly used inhalation anesthetics in veterinary medicine today. This study compared the anesthetic effects between isoflurane and sevoflurane in 17 calves undergoing surgery for placement of portal and jugular vein cannulas. Using a randomized control trial, calves were assigned to receive sevoflurane or isoflurane. Anesthesia was induced with xylazine and ketamine then maintained with the assigned inhalation anesthetic. Parameters for heart rate, respiratory rate, indirect blood pressures, oxygen hemoglobin saturation and end-tidal carbon dioxide were monitored and recorded during surgery. The anesthetic concentrations of the vaporizers were adjusted according to the calves' responses, e.g., blood pressure, eye position, respiratory and heart rate, to surgical stimulation. Upon discontinuation of the inhalation anesthetic, calves were placed in sternal recumbency and recovery variables including time to extubation, time to first movement, attempts to stand and time to standing were observed and recorded. Statistical analysis was performed using a two-sample t-test on the recorded variables. There were no statistical differences between isoflurane and sevoflurane for any of the parameters recorded during anesthetic maintenance or recovery. The results of this study showed a faster time to first movement and extubation, $3.38 \pm 1.85 \mathrm{~min}$ and $11.75 \pm 3.73 \mathrm{~min}$ for sevoflurane compared to $7.56 \pm 5.34 \mathrm{~min}$ and $15.56 \pm 8.69 \mathrm{~min}$ for isoflurane, respectively. Attempts to stand were $3.00 \pm 2.14$ for sevoflurane and $3.22 \pm 1.79$ for isoflurane. Though the time to standing during recovery was not statistically different between anesthetics, the values did indicate a quicker trend of recovery from sevoflurane. Both inhalation anesthetics produced comparable anesthetic qualities and there were no statistical differences between the parameters recorded during maintenance of anesthesia. On the basis of the reported results, sevoflurane and isoflurane are suitable inhalation anesthetics for use in calves. However, present cost of sevoflurane is a limiting factor for its use in food animals.
\end{abstract}

Keywords: Anesthesia, Sevoflurane, Isoflurane, Inhalation, Cattle

\section{INTRODUCTION}

Isoflurane and sevoflurane are the two most commonly used inhalation anesthetics in veterinary medicine today. Isoflurane exists as a clear nonflammable, halogenated methyl ethyl ether with a blood-gas partition coefficient of 1.46 (Steffey and
Mama, 2007; Stoelting, 1999). Sevoflurane is nonflammable, fluorinated methyl isopropyl ether with a lower blood-gas partition coefficient of 0.68 (Steffey and Mama, 2007; Stoelting, 1999). The blood-gas partition coefficient represents the partial pressure or solubility of an anesthetic between blood and gas at equilibrium. The higher the value of blood-gas partition coefficient, the 36849, USA Tel: 334-844-4490 
greater the solubility of an anesthetic in the blood and vice versa, the lower the value, the lower the blood solubility of the anesthetic. The substitution of a fluorine for chlorine in sevoflurane decreases its blood solubility and hence a lower blood-gas partition coefficient which allows a more rapid increase in alveolar anesthetic concentration during induction and a faster decrease during recovery, thus shorter anesthetic induction and recovery times (Johnson et al., 1998; Kazama and Ikeda, 1988; Mutoh et al., 1995; Steffey and Mama, 2007; Stoelting, 1999). In addition, a lower partition coefficient allows for a faster change of the anesthetic depth which greatly increases the safety of anesthesia.

The cardiovascular effects of sevoflurane are reportedly similar to isoflurane where both anesthetics cause dose dependent decreases in blood pressure, cardiac output and systemic vascular resistance in humans, dogs, horses, goats and sheep (Aida et al., 1996; Ebert et al., 1995; Hikasa et al., 1998; 2000; Mutoh et al., 1997). An increase in heart rate has been reported in dogs (Bernard et al., 1990; Ebert et al., 1995; Mutoh et al., 1997) whereas it remained unchanged in humans, sheep and goats (Ebert et al., 1995; Hikasa et al., 1998; 2000) during sevoflurane anesthesia. At 1 Minimum Alveolar Concentration (MAC), both sevoflurane and isoflurane cause a dose dependent decrease in respiratory rate and an increase in end-tidal partial pressures of carbon dioxide (PETCO2) in dogs, sheep and goats (Galloway et al., 2004; Hikasa et al., 1998; 2000; Johnson et al., 1998; Mutoh et al., 1997). Sevoflurane has the advantage of causing less airway mucosa irritation than isoflurane in humans, particularly during mask induction (Doi and Ikeda, 1993).

Sevoflurane has been reported to be a safe and effective inhalation anesthetic in adult cattle with a rapid and smooth recovery (Hikasa et al., 1994). However, because the high cost of sevoflurane, isoflurane is more frequently used in farm animal practice. The purpose of this study was to compare the anesthetic effects of isoflurane and sevoflurane in calves undergoing surgery for placement of portal and jugular vein cannulas.

\section{MATERIALS AND METHODS}

\subsection{Calves}

Seventeen healthy Holstein steer calves with a mean body weight of $279.94 \pm 36.06 \mathrm{~kg}$ were utilized in this study. The project was approved by the Auburn University Institutional Animal Care and Use Committee. The calves were anesthetized for surgical placement of portal and jugular vein cannulas for research purposes. Feed and water were withheld for 24 and $12 \mathrm{~h}$, respectively, prior to anesthesia to minimize the potential for bloat, regurgitation and aspiration pneumonia. All calves received $2.2 \mathrm{mg} / \mathrm{kg}$ of ceftiofur sodium (Naxcel ${ }^{\circledR}$, Pharmacia and Upjohn Co., Pfizer Inc., New York, NY) intravenously, prior to surgery. For induction of anesthesia and administration of fluid therapy during surgery, a 14 gauge, $140 \mathrm{~mm}$ catheter (Abbocath, Hospira, Inc., Lake Forest, IL) was placed aseptically in the right jugular vein before surgery.

\subsection{Induction and Anesthetic Equipment}

Anesthesia was induced with a bolus administration of $0.11 \mathrm{mg} / \mathrm{kg}$ of xylazine (AnaSed $\AA$, Lloyd Laboratories, Shenandoah, IA) and $2.2 \mathrm{mg} / \mathrm{kg}$ of ketamine (Ketaset ${ }^{\circledR}$, Fort Dodge Labs, Inc, Ft. Dodge, IA) intravenously via the jugular catheter. The calves were maintained in sternal recumbency for tracheal intubation and then placed in left lateral recumbency for the surgical procedure. Anesthesia was maintained with either isoflurane or sevoflurane in oxygen via a semiclosed circle breathing system. Calves were maintained on spontaneous ventilation, although a manual breath (sigh) by compressing the rebreathing bag was provided every 3 to $5 \mathrm{~min}$ at a pressure of $20 \mathrm{~cm}$ of $\mathrm{H} 2 \mathrm{O}$ to ensure appropriate ventilation. The anesthetic concentrations of the vaporizers were adjusted according to the calves' responses, e.g., blood pressure, eye position, respiratory and heart rate, to surgical stimulation. A balanced electrolyte solution (Veterinary Plasma-lyte A, Abbott Laboratories, North Chicago, IL) was administered continuously through the jugular catheter at a rate of 10 $\mathrm{mL} / \mathrm{kg} / \mathrm{h}$ for the duration of the anesthesia.

\subsection{Experimental Design and Surgical Procedure}

Calves were assigned at random to either isoflurane (Attane $^{\mathrm{TM}}$, Piramal Critical Care, Bethlehem, PA) or sevoflurane (SevoFlo ${ }^{\mathrm{TM}}$, Abbott Laboratories, Chicago, IL) groups. Portal vein cannulation was performed in all calves. Aseptic techniques were used during the laparotomy and placement of the portal vein cannulas. A $30-\mathrm{cm}$ abdominal incision was made at a location approximately $4 \mathrm{~cm}$ caudal to the 13 th rib and $5 \mathrm{~cm}$ ventral to the first lumbar vertebra. The skin incision followed the costal arch and the underlying muscle layers and peritoneum were incised individually to access the peritoneal cavity. The portal vein was identified in the omasal impression region between the left and quadrate lobes of the liver. Using blunt dissection, the hepatic lymph node was displaced in order to visualize and gain access to the portal vein. Using a $3 \mathrm{~cm}$, 16-gauge peel-away introducer needle, a 
16-gauge, $120 \mathrm{~cm}$ polyurethane catheter (Mila International, Inc., Erlanger, KY) was placed in the hepatic portal vein at the level of the porta hepatis. The tip of the catheter was positioned in close proximity to where the portal vein enters the liver permitting blood sampling from the entire splanchnic circulation. The portal catheter was maintained in place with adjustable sutures and suture wings placed along the lateral surface of the portal vein and the omentum surrounding the pancreas and duodenum. The catheter was exteriorized in the dorsolumbar region between the transverse processes of the 2nd and 3rd lumbar vertebra. A nylon purse-string suture secured the catheter at the exit site. The laparotomy incision was closed in routine fashion: with the peritoneum and transverse abdominal muscle closed together using \#2 chromic gut in a continuous pattern; internal and external abdominal oblique muscles closed using $\# 2$ chromic gut in a continuous pattern; and skin closed using \#2 Nylon in a Ford interlocking pattern.

\subsection{Data Collection and Measurements}

All parameters were collected using a Surgivet Advisor Monitor (Smiths Medical PM, Inc., Waukesha, WI). Heart Rate (HR), Respiratory Rate (RR), systolic, mean and diastolic arterial blood pressures (SAP, MAP and DAP, respectively), endtidal partial pressure carbon dioxide (PETCO2) and oxygen hemoglobin Saturation (SpO2) were monitored continuously and the values recorded at time 0 (baseline), which was immediately following induction with xylazine and ketamine and 10, 20, 40, 60 and 80 min during maintenance with isoflurane or sevoflurane. HR and Electrocardiogram (ECG) were monitored using a multi-functional electrocardiograph via hookup to Einthoven's triangle. SAP, MAP and DAP were measured by a non-invasive oscillometric cuff placed over the coccygeal artery at the base of the tail. RR was determined by movement of rebreathing bag and/or chest movement. The PETCO2 was measured by an infrared gas analysis with as ample line placed at the tip of the $\mathrm{Y}$ piece. The PETCO2 monitor is calibrated monthly. SpO2 was measured by means of a pulse oximetry placed on the lingual artery. The time to extubation, time to first movement, attempts to stand and time to standing were observed and recorded after discontinuation of the anesthetic.

\subsection{Statistical Analysis}

All data was analyzed for normality using a normality probability plot test using Minitab ${ }^{\circledR}$ (Minitab ${ }^{\circledR}$, Minitab Inc., State College, PA). A paired T

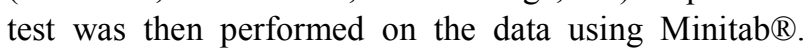
Values of $\mathrm{P}<0.05$ were considered significant.

\section{RESULTS}

All the parameters in this study were expressed as mean $\pm \mathrm{SD}$. All data analyzed fit the trend lines on the probability plot therefore it was considered normal. There were no significant differences for $\mathrm{HR}, \mathrm{RR}, \mathrm{SpO} 2$, PETCO2, SAP, MAP and DAP between isoflurane and sevoflurane during surgery (Table 1).

Table 1. Maintenance/monitored variables comparing isoflurane (Iso) vs. sevoflurane (Sevo) in Holstein calves undergoing surgery for portal cannula placement

\begin{tabular}{|c|c|c|c|c|c|c|}
\hline \multirow[b]{2}{*}{ Minutes of anesthesia } & \multicolumn{6}{|l|}{ ISO } \\
\hline & 0 & 10 & 20 & 40 & 60 & 80 \\
\hline \multicolumn{7}{|l|}{ Variable } \\
\hline Heart rate (beats/min) & $60.10 \pm 10.10$ & $71.22 \pm 4.87$ & $72.00 \pm 8.77$ & $68.33 \pm 9.01$ & $69.40 \pm 10.80$ & $72.00 \pm 13.70$ \\
\hline Respiratory rate (breaths/min) & $48.20 \pm 14.00$ & $46.10 \pm 13.40$ & $40.60 \pm 17.70$ & $37.70 \pm 18.20$ & $39.30 \pm 12.60$ & $40.00 \pm 7.94$ \\
\hline $\begin{array}{l}\text { Oxygen hemoglobin } \\
\text { saturation }(\% \mathrm{HbSat})\end{array}$ & $96.60 \pm 2.46$ & $95.60 \pm 3.95$ & $95.90 \pm 3.51$ & $94.80 \pm 6.41$ & $94.50 \pm 6.84$ & $95.63 \pm 4.44$ \\
\hline End-tidal $\mathrm{CO}^{2}(\mathrm{~mm} \mathrm{Hg})$ & $22.38 \pm 8.30$ & $30.00 \pm 7.45$ & $31.75 \pm 8.53$ & $35.80 \pm 13.00$ & $34.10 \pm 10.10$ & $35.30 \pm 12.50$ \\
\hline Systolic blood pressure (mm Hg) & $116.40 \pm 11.40$ & $104.30 \pm 13.10$ & $97.80 \pm 11.10$ & $103.30 \pm 15.50$ & $106.10 \pm 13.80$ & $112.10 \pm 10.40$ \\
\hline Mean blood pressure $(\mathrm{mm} \mathrm{Hg})$ & $91.80 \pm 20.10$ & $77.80 \pm 17.20$ & $69.56 \pm 8.00$ & $86.20 \pm 19.20$ & $91.90 \pm 16.70$ & $95.30 \pm 17.80$ \\
\hline Diastolic blood pressure (mm Hg) & $82.70 \pm 14.80$ & $68.30 \pm 16.40$ & $55.33 \pm 4.80$ & $67.20 \pm 16.00$ & $75.80 \pm 11.60$ & $75.11 \pm 8.68$ \\
\hline \multicolumn{7}{|l|}{ Sevo } \\
\hline \multicolumn{7}{|l|}{ Variable } \\
\hline Heart rate (beats/min) & $63.1 \pm 16.30$ & $72.38 \pm 8.98$ & $70.75 \pm 7.57$ & $69.75 \pm 07.55$ & $67.00 \pm 8.00$ & $67.70 \pm 10.00$ \\
\hline Respiratory rate (breaths/min) & $34.60 \pm 21.20$ & $35.00 \pm 18.90$ & $46.71 \pm 7.95$ & $39.30 \pm 10.80$ & $42.86 \pm 8.05$ & $44.33 \pm 6.43$ \\
\hline $\begin{array}{l}\text { Oxygen hemoglobin } \\
\text { saturation }(\% \mathrm{HbSat})\end{array}$ & $94.88 \pm 4.45$ & $97.00 \pm 2.07$ & $96.13 \pm 3.60$ & $98.38 \pm 1.19$ & $96.38 \pm 2.39$ & $98.00 \pm 1.00$ \\
\hline End-tidal $\mathrm{CO}^{2}(\mathrm{~mm} \mathrm{Hg})$ & $31.10 \pm 13.00$ & $37.38 \pm 9.35$ & $30.90 \pm 11.80$ & $38.50 \pm 10.10$ & $43.25 \pm 7.67$ & $43.33 \pm 7.57$ \\
\hline Systolic blood pressure (mm Hg) & $113.40 \pm 12.10$ & $96.50 \pm 18.40$ & $93.30 \pm 16.70$ & $106.10 \pm 14.00$ & $117.40 \pm 15.10$ & $120.60 \pm 14.40$ \\
\hline Mean blood pressure (mm Hg) & $89.00 \pm 10.30$ & $75.90 \pm 19.10$ & $73.90 \pm 14.90$ & $85.10 \pm 13.40$ & $98.60 \pm 18.50$ & $96.00 \pm 8.76$ \\
\hline Diastolic blood pressure (mm Hg) & $81.40 \pm 15.10$ & $64.50 \pm 19.80$ & $58.40 \pm 12.90$ & $69.30 \pm 10.90$ & $80.60 \pm 20.50$ & $82.50 \pm 16.80$ \\
\hline
\end{tabular}


Table 2. Anesthesia recovery data comparing isoflurane (Iso) vs. Sevoflurane (Sevo) in Holstein calves undergoing surgery for portal cannula placement

\begin{tabular}{lccc}
\hline & ISO & SEVO & *SEVO minus calf 70 \\
\hline Time to first movement (minutes) & $7.56 \pm 5.34$ & $3.38 \pm 1.85$ & $3.57 \pm 1.90$ \\
Time to extubation (minutes) & $15.56 \pm 8.69$ & $11.75 \pm 3.73$ & $12.57 \pm 3.15$ \\
Time to standing (minutes) & $47.20 \pm 29.00$ & $53.90 \pm 61.90$ & $32.70 \pm 17.30$ \\
Attempts to stand (number of attempts) & $3.22 \pm 1.79$ & $3.00 \pm 2.14$ & $2.29 \pm 0.75$ \\
\hline
\end{tabular}

* Corrected sevoflurane data minus calf \#70. Calf 70 removed from data for post-surgical hemorrhage complication.

There was no statistical difference for the time to standing during recovery between isoflurane and sevoflurane (Table 2). The results of this study showed a faster time to first movement $(3.38 \pm 1.85 \mathrm{~min}$ for sevoflurane vs. $7.56 \pm 5.34 \mathrm{~min}$ for isoflurane) (Table 2) and time to extubation $(11.75 \pm 3.73 \mathrm{~min}$ for sevoflurane vs. $15.56 \pm 8.69 \mathrm{~min}$ for isoflurane) (Table 2). Once the anesthetic was discontinued, the number of attempts to stand was $3.00 \pm 2.14$ for sevoflurane and $3.22 \pm 1.79$ for isoflurane (Table 2). Though the time to standing during recovery was not statistically different between isoflurane and sevoflurane $(47.20 \pm 29.00 \mathrm{~min}$ for isoflurane vs. $53.90 \pm 61.90 \mathrm{~min}$ for sevoflurane) (Table 2), with sevoflurane having a longer time to standing. These values are misleading due to an abnormally long recovery in one calf (calf \#70) due to a post-surgical hemorrhage complication. Removal of calf 70 improved the standing time to $32.70 \pm 17.30 \mathrm{~min}$ for sevoflurane (Table 2). These values did indicate a trend that recovery from sevoflurane was faster than isoflurane as supported by the faster time to first movement, time to extubation and standing time (Table 2).

\section{DISCUSSION}

Isoflurane is the most widely used inhalation anesthetic in veterinary practice today, with sevoflurane gaining popularity especially in small animals. Clinically, the lower blood-gas partition coefficient of sevoflurane has been shown in dogs to result in a more rapid increase in alveolar anesthetic concentration during induction and decrease during recovery, as reflected in a faster induction and quicker recovery (Johnson et al., 1998; Kazama and Ikeda, 1988; Mutoh et al., 1995). Sevoflurane has been reported to be an effective inhalation anesthetic in adult cattle with a rapid and smooth recovery (Hikasa et al., 1994).

The cardiovascular effects of sevoflurane are reportedly similar to isoflurane where both anesthetics cause dose dependent decreases in blood pressure, cardiac output and systemic vascular resistance in humans, dogs, horses, sheep and goats (Aida et al., 1996;
Ebert et al., 1995; Hikasa et al., 1998; 2000; Mutoh et al., 1997; Steffey and Mama, 2007; Stoelting, 1999). In awake cattle, normal HR and MAP have been reported to range from $60-80$ beats per min with a pressure range of 90-140 mm Hg, respectively (Muir et al., 2007). Results from this study showed no statistical differences in SAP, MAP or DAP between the two anesthetics. Between 0 and $20 \mathrm{~min}$, following the administration of isoflurane and sevoflurane anesthetics, the SAP, MAP and DAP all decreased. This is thought to be due to absence of surgical stimulation and the wearing off of the induction drugs, xylazine and ketamine. The surgical procedure started at approximately $30 \mathrm{~min}$ following the induction drugs. At $40 \mathrm{~min}$, post induction, both anesthetics showed similar increases in SAP, MAP and DAP, which is believed to be a response to the surgical stimulation. Close analysis of the data in this study shows that the values of blood pressures for both anesthetics were similar throughout the surgery. A decrease in blood pressure, cardiac output and systemic vascular resistance can cause an increase in HR due to baroreceptor reflex (Stoelting, 1999). Several studies indicated an increase in heart rate in dogs (Bernard et al., 1990; Ebert et al., 1995; Mutoh et al., 1997) whereas heart rate remained unchanged in humans, sheep and goats (Ebert et al., 1995; Hikasa et al., 1998; 2000) during sevoflurane anesthesia. In dogs, it has been suggested that the differences in vagolytic activity of the inhalation anesthetics is responsible for the increase in HR observed (Steffey and Mama, 2007). In horses HR increased as the concentration of sevoflurane increased above $1 \mathrm{MAC}$, but the differences were not significant (Aida et al., 1996). In the present study, heart rate remained steady throughout the surgery and there was no statistical differences noted between sevoflurane and isoflurane. No hematological or serum chemistry profile analysis were performed in this study. However, no significant differences were observed in the blood work of sheep (Hikasa et al., 2000) receiving isoflurane or sevoflurane.

Sevoflurane and isoflurane have been reported to cause similar dose-dependent respiratory effects 
characterized by a decrease in RR and an increase in PETCO2 in dogs, horses and goats when maintained at 1 MAC (Aida et al., 1996; Galloway et al., 2004; Hikasa et al., 1998; Johnson et al., 1998; Mutoh et al., 1997). In ruminants, positioning in lateral recumbency (Fujimoto and Lenehan, 1985) and bloating due to continuous ruminal fermentation during anesthesia has been shown to impair ventilation (Lin and Pugh, 2012). These factors may contribute to higher levels of PETCO2, respiratory acidosis and lower $\mathrm{SpO} 2$. In the present study, neither isoflurane nor sevoflurane caused significant effects on RR nor was there a significant difference on RR between the two anesthetics. Although an increase in PETCO2 levels were observed during surgery with sevoflurane and isoflurane calves with the values for sevoflurane being slightly higher than that for isoflurane, these values were not statistically significant. These findings in calves are similar to those reported in sheep (Hikasa et al., 2000). The SpO2 concentration was slightly higher for sevoflurane than isoflurane but both were within normal reference ranges.

In previous studies, the duration of recovery to standing has always been shorter with sevoflurane than with isoflurane in horses, sheep and dogs (Hikasa et al., 2000; Johnson et al., 1998; Matthews et al., 1998). Recovery quality of sevoflurane has been reported as comparable to or better than isoflurane in dogs and horses (Johnson et al., 1998; Matthews et al., 1998). Although this study found no statistical difference in the time to first movement, time to extubation, time to standing or attempts to stand between the two anesthetics, sevoflurane anesthetized calves appeared to awaken from anesthesia faster as indicated by the less time to first movement and extubation.

Calf \#70, anesthetized with sevoflurane, had an abnormally prolonged recovery time as a result of a dislodged portal vein cannula, which led to excess blood loss and muscle weakness. A balanced electrolyte solution (Veterinary Plasma-lyte A, Abbott Laboratories, North Chicago, IL) was administered to offset the blood loss and the calf recovered and stood in $197 \mathrm{~min}$ after removal of the endotracheal tube. When calf \#70 is excluded from the data, time to standing and attempts to stand drastically improved over isoflurane. Treating calf \#70 as an outlier and removing it from the sevoflurane group and statistical analyzed data, sevoflurane calves had faster recovery time to standing and less attempts to stand $(32.70 \pm 17.30 \mathrm{~min}$ and $2.29 \pm 0.75$ attempts) than isoflurane calves $(47.20 \pm 29.00 \mathrm{~min}$ and $3.22 \pm 1.79$ attempts), though the differences were not statistically significant (Table 2).

Sevoflurane has a number of advantages over isoflurane. Sevoflurane has a lower blood-gas partition coefficient of 0.68 compared to 1.46 for isoflurane (Steffey and Mama, 2007; Stoelting, 1999). This lower blood solubility allows for a rapid induction of and recovery from anesthesia and rapid anesthetic depth changes, which greatly increases the safety of anesthesia for large animal patients. Possible disadvantages of sevoflurane over isoflurane include the possibility of nephrotoxicity and higher cost. Sevoflurane has also been reported to cause less airway mucosa irritation than isoflurane in humans, particularly if mask induction is used (Doi and Ikeda, 1993).

Dry $\mathrm{CO} 2$ absorbents can degrade isoflurane to carbon monoxide $(\mathrm{CO})$ and sevoflurane to a vinyl ether (fluoromethyl-2,2-difluoro-1-9trifluoromethyl) also known as compound A (Higuchi et al., 2000; Kenna and Jones, 1995; Stoelting, 1999). Potassium hydroxide $(\mathrm{KOH})$ and sodium hydroxide $(\mathrm{NaOH})$, primary components of $\mathrm{CO} 2$ absorbents, have been identified as major determinants in the production of $\mathrm{CO}$ and compound A (Higuchi et al., 2000). Rats appear to be especially sensitive to the nephrotoxic effects of compound A, while humans are at relatively low risk (Kenna and Jones, 1995). Dogs anesthetized with 4\% to $5 \%$ sevoflurane in $500 \mathrm{~mL}$ per min oxygen (O2) flow for $3 \mathrm{~h}$ sessions did not produce concentrations of compound A associated with renal toxicity, as evidenced by changes in serum biochemistry and urinalysis (Wallin et al., 1975). When using a rebreathing system, the risk of compound A induced nephrotoxicity can be reduced by using an $\mathrm{O} 2$ flow rate higher than the animal's metabolic requirement in order to dilute and flush out compound A from the breathing system (Muir and Gadawski, 1998; Stoelting, 1999). The calves in this study were maintained on a semi-closed rebreathing system using an $\mathrm{O} 2$ flow rate that is three times the $\mathrm{O} 2$ metabolic requirement. Other options include using newer $\mathrm{CO} 2$ absorbents, Amsorb ${ }^{\circledR}$ (Armstrong Medical, Coleraine, Northern Ireland), which contains neither $\mathrm{KOH}$ nor $\mathrm{NaOH}$, Drägersorb 800 plus ${ }^{\circledR}$ (Dräger, Luebeck, Germany) and Medisorb ${ }^{\circledR}$ (Datex-Ohmeda, Bromma, Sweden) which contain minimal amounts of $\mathrm{NaOH}(1-2 \%)$ and trace amounts of $\mathrm{KOH}(0.003 \%)$ (Higuchi et al., 2000).

The higher cost of Sevoflurane is also a disadvantage, especially in production animals. At present, sevoflurane cost seven times that of isoflurane per $\mathrm{mL}$. Sevoflurane also has a lower potency when 
compared to isoflurane. The MAC reported for sevoflurane is $2.25 \%$ compared to $1.5 \%$ for isoflurane in sheep and goats (Antognini and Eisele, 1993; Mohamadnia et al., 2008), which is $0.75 \%$ or 1.67 times higher MAC requirement for sevoflurane than isoflurane. This higher MAC, for anesthetic maintenance plus the higher cost per $\mathrm{mL}$ for the anesthetic obviously lead to a higher overall anesthesia cost. In production animals, added costs affect producer profitability, which are already small on a per animal basis.

\section{CONCLUSION}

This study indicates that sevoflurane and isoflurane are both suitable inhalation anesthetics for the maintenance of general anesthesia in Holstein calves undergoing elective surgery. The results of the study showed no statistically significant differences in cardiovascular or respiratory effects when comparing sevoflurane to isoflurane, which are consistent with previous reports in dogs, sheep, goats, humans and horses (Aida et al., 1996; Ebert et al., 1995; Hikasa et al., 1998; 2000; Matthews et al., 1998; Mutoh et al., 1997). Attempts to stand and recovery times appeared to occur faster for sevoflurane upon review of the data, although not statistically significant. Sevoflurane appears to have a slight advantage over isoflurane in the recovery phase of anesthesia but, at present, that advantage may be overcome by the higher cost of the drug in production animals.

\section{ACKNOWLEDGEMENT}

Funding for this study was provided by Auburn University College of Veterinary Medicine Interdisciplinary Research and Equipment Grant.

\section{REFERENCES}

Aida, H., Y. Mizuno, S. Hobo, K. Yoshida and T. Fujinaga, 1996. Cardiovascular and pulmonary effects of sevoflurane anesthesia in horses. Vet. Surg., 25: 164-170. PMID: 8928394

Antognini, J.F. and P.H. Eisele, 1993. Anesthetic potency and cardiopulmonary effects of enflurane, halothane and isoflurane in goats. Lab Anim. Sci., 43: 607-610. PMID: 8158989

Bernard, J.M., P.F. Wouters, M.F. Doursout, B. Florence and J.E. Chelly et al., 1990. Effects of sevoflurane and isoflurane on cardiac and coronary dynamics in chronically instrumented dogs. Anesthesiol., 72: 659-62. PMID: 2321783
Doi, M. and K. Ikeda, 1993. Airway irritation produced by volatile anaesthetics during brief inhalation: Comparison of halothane, enflurane, isoflurane and sevoflurane. Can. J. Anaesth., 40: 122-126. PMID: 8443850

Ebert, T., C. Harkin and M. Muzi, 1995. Cardiovascular responses to sevoflurane: A review. Anesth. Analg., 81: S11-S22. PMID: 7486143

Fujimoto, J.L. and T.M. Lenehan, 1985. The Influence of body position on the blood gas and acid-base status of halothane-anesithetized sheep. Vet. Surg., 14: 169-172. DOI: $\quad 10.1111 /$ j.1532950X.1985.tb00855.X

Galloway, D.S., J.C.H. Ko, H.F. Reaugh, R.E. Mandsager and M.E. Payton et al., 2004. Anesthetic indices of sevoflurane and isoflurane in unpremedicated dogs. J. Am. Vet. Med. Assoc., 225: 700-704. DOI: 10.2460/javma.2004.225.700

Higuchi, H., Y. Adachi, S. Arimura, M. Kanno and T. Satoh, 2000. Compound A concentrations during low-flow sevoflurane anesthesia correlate directly with the concentration of monovalent bases in carbon dioxide absorbents. Anesth. Analg., 91: 434439. PMID: 10910864

Hikasa, Y., K. Okuyama, T. Kakuta, K. Takase and S. Ogasawara, 1998. Anesthetic potency and cardiopulmonary effects of sevoflurane in goats: Comparison with isoflurane and halothane. Can. J. Vet. Res., 62: 299-306. PMID: 9798097

Hikasa, Y., K. Saito, K. Takase and S. Ogasawara, 2000. Clinical, cardiopulmonary, hematological and serum biochemical effects of sevoflurane and isoflurane anesthesia in oxygen under spontaneous breathing in sheep. Small Ruminant Res., 36: 241-249. PMID: 10781740

Hikasa, Y., K. Takase, K. Kondou and S. Ogasawara, 1994. Sevoflurane anesthesia following administration of atropine-guaifenesin-thiopental in spontaneous-breathing adult cattle. J. Vet. Med. Sci., 56: 700-708. PMID: 7948408

Johnson, R.A., E. Striler, D.C. Sawyer and D.B. Brunson, 1998. Comparison of isoflurane with sevoflurane for anesthesia induction and recovery in adult dogs. Am. J. Vet. Res., 59: 478-481. PMID: 9563634

Kazama, T. and K. Ikeda, 1988. Comparison of MAC and the rate of rise of alveolar concentration of sevoflurane with halothane and isoflurane in the dog. Anesthesiology, 68: 435-437. PMID: 3345000

Kenna, J.G. and R.M. Jones, 1995. The organ toxicity of inhaled anesthetics. Anesth. Analg., 81: s51-s66. PMID: 7486149 
Lin, H.C. and D.G. Pugh, 2012. Anesthetic Management. In: Sheep and Goat Medicine, Pugh, D.G. and N. Baird (Eds.), Elsevier Health Sciences, Maryland Heights, Mo., ISBN-10: 1437723543 pp: 415.

Matthews, N.S., S.M. Hartsfield, D. Mercer, M.H. Beleau and A. MacKenthun, 1998. Recovery from sevoflurane anesthesia in horses: Comparison to isoflurane and effect of postmedication with xylazine. Vet. Surg., 27: 480-485. PMID: 9749520

Mohamadnia, A.R., G. Hughes and K.W. Clarke, 2008. Maintenance Maintenance of anaesthesia in sheep with isoflurane, desflurane or sevoflurane. Vet. Rec., 163: 210-215. PMID: 18708654

Muir, W.W. and J. Gadawski, 1998. Cardiorespiratory effects of low-flow and closed circuit inhalation anesthesia, using sevoflurane delivered with an incircuit vaporizer and concentrations of compound A. Am. J. Vet. Res., 59: 603-608. PMID: 9582964

Muir, W.W., J.A.E. Hubbell and R.M. Bednarski, 2007. Handbook of Veterinary Anesthesia. 4th Edn., Mosby Elsevier's, Edinburgh, ISBN-10: 0323046789, pp: 643.

Mutoh, T., R. Nishimura, H.Y. Kim, S. Matsunaga and N. Sasaki, 1997. Cardiopulmonary effects of sevoflurane, compared with halothane, enflurane and isoflurane, in dogs. Am. J. Vet. Res., 58: 885890. PMID: 9256976
Mutoh, T., R. Nishimura, H.Y. Kim, S. Matsunaga and T. Kadosawa et al., 1995. Rapid inhalation induction of anesthesia by halothane, enflurane, isoflurane and sevoflurane and their cardiopulmonary effects in dogs. J. Vet. Med. Sci., 56: 1007-1013. PMID: 872003

Steffey, E.P. and K.R. Mama, 2007. Inhalation Anesthetics. In: Veterinary Anesthesia and Analgesia, Tranquilli, W.J., J.C. Thurmon and K.A. Grimm (Eds.), Wiley, Ames, IA., ISBN-10: 0781754712, pp: 355-393.

Stoelting, R.K., 1999. Inhaled Anesthetics. In: Pharmacology and Physiology in Anesthetic Practice, Percy, R.C. (Ed.), Lippincott-Raven, Philadelphia, pp: 36-76.

Wallin, R.F., B.M. Regan, M.D. Napoli and I.J. Stern, 1975. Sevoflurane: A new inhalational anesthetic agent. Anesth. Analg., 54: 758-766. PMID: 1239214 